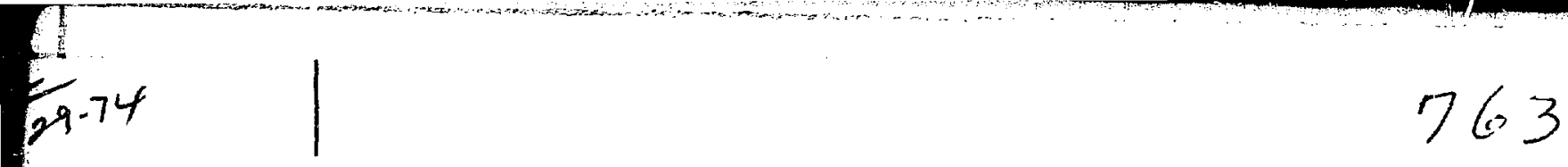

LA-5544-PR

Progress Report

Performance of Multiple HEPA Filters Against Plutonium Aerosols

July 1 through December 31, 1973 
This report presents the status of the Performance of Multiple HEPA Filters Against Plutonium Aerosols at LASL. The previous quarterlies in this series, unclassified, are:
LA-5012-PR
LA-5349-PR

LA-5170-PR

In the interest of prompt distribution, this progress report was not edited by the Technical Information staff.

Printed in the United States of America. Available from National Technical information Service

U. S. Department of Commerce

5285 Port Royal Road

Springfield, Virginia 22151

Price: Printed Copy $\$ 4.00$ Microfiche $\$ 1.45$ 


\section{Performance of Multiple HEPA Filters Against Plutonium Aerosols}

July 1 through December 31, 1973

by

Harry J. Ettinger, Project Manager

John C. Elder

Manuel Gonzales

This report was prepared as an account of work
sponsored by the United States Government. Neither
the United States nor the United States Atomic Energy
Cominission, nor any of their employees, nor any of
their contractors, subcontractors, or their employees,
makes any warranty, expsess or implied, or assumes any
legal liability or responsibility for the accuracy, com.
pleteness or usefulness of any information, opparatus,
product or process disclosed, or represents that its use
would not infringe privatily owned rights. 
PERFORMANCE OF MULTIPLE HEPA FILTERS

AGAINST PLUTONIUM AEROSOLS

DY

Harry J. Ettinger, Project Manager, John C. Elder, and Manuel Gonzales

ABSTRACT

A multiple HEPA filter system has been evaluated using laboratory produced plutonium aerosols with size characteristics substantially smaller than the $0.7-\mu \mathrm{m}$ activity median aerodyramic diameter (amad) attained during the previous reporting period. Improved cierosol preparation by centrifugal ball milling of $238_{\mathrm{PuO}_{2}}$ and subsequent nebulization of water suspensions has produced challenge aerosols with amad's in the range 0.3 to $0.5 \mu \mathrm{m}$, and in concentrations high enough for testing three HEPA filters in series. These aerosols contain 108 to 308 of total activity in the aerodynamic size range below $0.22 \mu \mathrm{m}$, allowing efficiency measurement of the first two HEPA filters in series for this size interval. Efficiencies of HEPA \#1 and HEPA \#2 have exceeded $99.98 \%$ for all sizes of plutonium aerosol, including the intervals 0.12 to $0.22 \mu \mathrm{m}$ and $<0.12 \mu \mathrm{m}$. Efficiency of HiEPA \#3 was 99.758 expressed as the average of eight runs. Tests now in progress are expected to show that several of the lowest HEPA \#3 efficiencies (99.58) were artifacts caused by low level counting problems, and that the actual HEPA \#3 efficiency will exceed $99.75 \%$.

Limited field sampling at Locatior 00 is continuing with spectrum analysis of impactor samples ${ }^{\text {roviding }}$ sevarate particle size characteristics of ${ }^{238} \mathrm{Pu}$ and ${ }^{239} \mathrm{Pu}$ mixed in the process ventilation system. In conjunction with this field sampling, several coating materials are being applied to impactor plates to further evaluate particle rebound effects.

Calculated exposures to alpha radiation due to $\mathrm{Pu}$ particles collected in the HEPA filter media are comparable to exposures causing observable effects in glass containers. Possible loss of efficiency in existing HEPA filters exposed to $238 \mathrm{PuO}_{2}$ particles for 8 to 10 months will be investigated experimentally.

\section{SUMMARY}

Major progress in evaluating the performance of three HEPA filters in series against plutonium aerosols consisted of reducing the $\mathrm{Pu}$ aerosol particle size below the 0.7- $\mu \mathrm{m}$ activity median aerodynamic diameter (amad) attained during the previous reporting period, and testing HEPA filters with these aerosols to define efficiency for particles as sma 1 as 0.12 to $0.22 \mu \mathrm{m}$. Centrifugal ball milling and subsequent nebulization of a ${ }^{238} \mathrm{PuO}_{2}$ water suspension have yielded aerosols with amad's primarily in the range 0.3 to $0.5 \mathrm{\mu m}$, and in concentrations high enough for testing three HEPA filters 
in series. These aerosols contain 10 to $30 \%$ of the total activity in the size range under $0.22 \mu \mathrm{m}$, which is sufficient to test two HEPA filters in series for this size fraction. The milling procedures appear to be more efficient but much of the gain in size reduction is lost due to re-agglomeration which occurs either by direct contact of the particles while in solution, or by the nebulizers trapping several small particles in one water droplet, and maintaining this agglomerate during subsequent drying of the droplet.

HEPA filter efficiencies against plutonium particles in these size ranges have been in excess of 99.988 for the first two HEPA filters. Extremely low count rates ( $\approx 0.01$ cps) downstream of the third HEPA, resulting in poor overall counting statistics at this sampiing point, make efficiency measurements for the third HEPA filter difficult to precisely define. Some recent runs with higher aerosol concentrations which improve statistical measurements have resulted in efficiencies for HEPA \#3 of 99.98 or better, but the average of eight tests indicates an efficiency of 99.758 . Efficiencies of the first two HEPA filters against all $\mathrm{Pu}$ aerosol size ranges, including 0.12 to $0.22 \mu \mathrm{m}$ and $<0.12 \mu \mathrm{m}$, have been above 99.998 .

Spectrum analysis of impactor samples taken from process ventilation ducts at Location $00^{1}$ is providing particle size characteristics of ${ }^{238} \mathrm{Pu}$ and ${ }^{239} \mathrm{Pu}$ aerosols produced by separate processes but mixed in the ventilation system. In conjunction with this field sampling, several coating materials have been applied to impactor plates to further eviluate particle rebound effects. Definite results in both areas are expected in the next report period.

Investigation of alpha radiation effects on glass indicates estimated exposure in the HEPA filter media are comparable to exposures causing observable effects in glass containers. Efficiency testing of existing HEPA filters exposed to ${ }^{238} \mathrm{PuO}_{2}$ particles for 8 to 10 months is scheduled.

II. FXPERIMENTAL TEST PROGRAM

Prior to this reporting period, ${ }^{238} \mathrm{PuO}_{2}$ test aerosols were generated by suspending dry ball-milled powder in water, adding an anionic surfactant, ultrasonically agitating the suspension, and aerosolizing with a modified Retec nebulizer. Minimum aerosol activity median aerodynamic diameter (amad) attained by this method was approximately $0.7 \mu \mathrm{m}$, significantly larger than the $0.1-\mu \mathrm{m}$ amad measured at Location $11^{1}$ in the field sampling program. Several avenues of particle size reduction using essentially the same experimental system and techniques for sampling and generation were evaluated in order to define the performance of HEPA filters for particles as small as $0.1 \mu \mathrm{m}$. This part of the progress report details those techniques used to generate these smaller test aerosols, modifications to the experimental system to characterize these smaller particle sizes, and the performance of HEPA filters under these test conditions.

\section{A. Aerosol Size Reduction}

A new Geoscience centrifugal ball mill was used to mill various batches of ${ }^{238} \mathrm{PuO}_{2}$ for varying time intervals. To attain similar particle size reductions with this mill, shorter milling times were anticipated because of higher rate of energy input. Milling was carried out using a carrier liquid which was expected to reduce agglomeration. Early wet milling procedures utilized ethanol, but high pressures generated within the mill enclosure necessitated a change to water as the carrier liquid. Additional problems were encountered as a result of alpha activity breaking down the water to $\mathrm{H}_{2}, \mathrm{O}_{2}$, and $\mathrm{H}_{2} \mathrm{O}_{2}$, again creating high pressures and explosive mixtures within the mill jar. A continuously vented mill enclosure was developed and pressure problems were eliminated. The new milling procedures have yielded aerosol amad's ranging from $0.31 \mu \mathrm{m}$ to $0.66 \mathrm{\mu m}$ for milling times ranging from 
44 to 1.19 hours. Though not reaching the desired $0.1-\mu \mathrm{m}$ amad, these size distributions do yield 10 to 30 of the material in the size range of interest, i.e., <0.22 $\mathrm{\mu m}$.

An aliquot of the same starting material was also heat treated at $1525^{\circ} \mathrm{C}$ for 17 hours and held at $1100^{\circ} \mathrm{C}$ for 6 hours to restore the crystalline structure of the $238_{\mathrm{PuO}_{2}}$ which undergoes self-radiation damage when stored for long periods. This heat treatment was attemptied to improve the fracturability of the material by ball milling. Particle sizes attained by tinis method were comparable to those previously described but did not provjde any significant size reduction.

Chemical precipitation often used in plutonium conversion processes was considered a promising source of fine plutonium parti-cles. Precipitation of the ${ }^{238} \mathrm{Pu}(\mathrm{OH})_{4}$ yields extremely small particles which upon heating leaves ${ }^{238} \mathrm{PuO}_{2}$ in cake form free of inert salts. This material was milled for 17 hours and generated by the Retec nebulizer with a $0.015^{\prime \prime}$ jet and agaj.n with 0.021 " jet. The

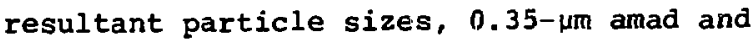
0.43- $\mu \mathrm{m}$ amad, respectively, did not show an overall improvement in size reduction compared to the original material.

Several reasons for below optimum generation from the Retec nebulizer were considered. These included: (1) the nebulizers were generating droplets large enough to contain several particles which joined into a single agglomerate as the droplet aried and (2) high particle concentration in the suspensions allowed extensive agglomeration which could not be offset by ultrasonic agitation prior to generation. The latter problem was examined early in the program without significant improvement in particle size at concentrations useful in testing three HEPA stages.

Various experiments were devised to test the former concern. One test involved using smaller jet sizes at the same air pressure to increase the velocity at which the atomized suspension contacts the impaction ball, thus breaking up the water droplets into smaller droplets. This same effect was also tested by generating with an unmodified generator at higher generating air pressure. Although a slight decrease in the overall particle size distribution was noted when the smaller jet diameter was used, the reduction was not significant enough to warrant routine use. Aerosol concentration was reduced by a factor of three by both of the above procedires, an effect that could not be tolerated if three HEPA filters in series are to be tested.

In fuz:ther attempts to reduce the water droplet size produced by the Retec nebulizer, a baffle plate was installed at the outlet to the nebulizer to remove the larger dropjets by impaction and allow the smaller ones to pass. Although some improvement was roted, the water collected at these baffle plates had no clear runoff area, and this water was re-entrained in the aerosol stream, resulting in significant quantities of water being ejected into the main duct and running down to HEPA filter \#1. Aerosol concentration was also markedly reauced. At the conclusion of these tests, it was decided to omit any baffles and to revert to the jet diameter provided by the unmodified Retec nebulizer. The result was a finer aerosol at a slightly lower concentration. The possibility that grinding actuallyt produces smaller individual particles, but agglomeration immediately prior to or during aerosol generation increased the apparent particle size was investigated. Airborne samples from a very dilute generator solution collected and transferred to EM grids ${ }^{2}$ indicated the presence of discrete particles which are very small (about $0.1-0.2 \mathrm{\mu m}$ aerodynamic diameter) with few doublets. Airborne samples were collected from two subsequent efficiency test runs (0.48- $\mu \mathrm{m}$ anad) and transferred to EM grids to check agglomeration of the aerosolized material. These electron micrographs showed that even the very small particles are made up of several smaller individual particles (Figure 1). 
This points to our inability to break up the agglomerates prior to nebulization, or to the limitation imposed by trapping more than one discrete $P u$ particle within a single aerosolized droplet.

B. Aerosol Sampling and Analysis Techniques

The sampling system ahead of each HEPA stage has been modified to allow impactor sampling at $1.42 \times 10^{-3} \mathrm{~m}^{3} / \mathrm{s}(3.0 \mathrm{cfm})$. As suggested by $\mathrm{Hu}^{3}$ and previously utilized in the field sanpling program, 4 operation of these impactors at higher flow rates shifts the effective range of particle size classification downward to include the lower limit of the range of interest $(0.1 \mu \mathrm{m})$. Effective cutoff diameters for $1.42 \times 10^{-3}$ $\mathrm{m}^{3} / \mathrm{s}(3.0 \mathrm{cfm})$ were calculated and compared with Hu's experimental results (Table I).

\section{TABLE I}

EFFECTIVE CUTOFF LIAMETERS (AERODYNAMIC) OF ANDERSEN IMPACTOR OPERATED AT $1.42 \times 10^{-3} \mathrm{~m}^{3} / \mathrm{s}(3.0 \mathrm{cfm})$

\begin{tabular}{ccc} 
Stage No. & $\frac{\text { Calcuiated }}{\text { Experimental }}{ }^{3}$ \\
\hline 0 & $5.4 \mu \mathrm{m}$ & - \\
1 & 3.4 & - \\
2 & 2.3 & $2.4 \mu \mathrm{m}$ \\
3 & 1.5 & 1.5 \\
4 & 0.96 & 0.9 \\
5 & 0.44 & 0.4 \\
6 & 0.22 & 0.17 \\
7 & 0.12 & -
\end{tabular}

As stated in the previous report, 4 good agreement exists between calculated and experimental effective cutoff diameter (ECD), and the calculated ECD's provide adequate estimates of the actual ECD's for stages 0 , 1 , and 7 where experimental data are lacking. System modifications consisted of (1) larger sampling probes to maintain isokinetic conditions, (2) individual hig̣h capacity pumps, and (3) larger backup membrane filter (MF) holders. Sampling procedure remained essentially the same as reported earlier except for extension of sampling time to 120 minutes to improve the count statistics for samples obtained downstream of HEPA filter $\# 3$.

\section{c. Overall HEPA Filter Efficiencies}

Several runs have been made using these smaller aerosols and operating the Andersen impactor at a flow rate of $1.5 \times 10^{-3} \mathrm{~m}^{3} / \mathrm{s}$ (3.2 cfm) to characterize aerosol fractions as small as 0.12 to $0.22 \mu \mathrm{m}$, and $<0.12 \mu \mathrm{m}$. Eight of 11 runs have been analyzed and these data are detailed in Table II and summarized in Table III. Three other runs are undergoing analysis or being held for Rn-Th decay.

Overall HEPA filter efficiencies for the first and second stages were all well within minimum criteria guidelines, with the minimum measured efficiency for each of the first two filters in series of $>99.98 \%$. HEPA filter $\$ 3$ in the series shows an average efficiency of $99.75 \%$, with a minimum efficiency of 99.508, significantly lower than HEPA $\$ 1$ or $\$ 2$. However, we believe these lower efficiencies are an artifact, and can be attributed to poor count statistics at sampler \#4 downstream of HEPA \#3. Several of the more recent tests employed higher initial aerosol concentrations to increase the challenge aerosol to HEPA filter \#3. Efficiencies for these tests are higher, and well within minimum criteria guidelines. Tests are also in progress using HEPA filters with quality control test data indica$t$ ing efficiencies close to but not less than 99.978. We expect these will also improve count statistics for the sampler downstream of HEPA $\$ 3$.

Table II also shows that aerosol size distributions do not change significantly with sub: squent filter stages, an observation noted with larger aerosols used in previous runs. 1 The $\sigma_{g}$ is decreased somewhat, indicating an aerosol with a narrower size range downstream of successive HEPA filters. However, these minor aerosol size variations suggest that the aerosol challenging the third HEPA filter is comparable to hat for the second HEPA, and filter performance for these filters should be the same. 
TABLE II

HEPA FILTER EFFICIENCY

\begin{tabular}{|c|c|c|c|c|c|}
\hline Run & $\begin{array}{l}\text { HEPA } \\
\text { Filter } \\
\text { stage } \\
\end{array}$ & $\begin{array}{l}\text { Pluto } \\
\text { amad (1 }\end{array}$ & $\begin{array}{c}\text { Aerosol } \\
\sigma_{g} \\
\end{array}$ & $\begin{array}{c}\text { Activity } \\
\text { Concentraţions } \\
\text { dps } / \mathrm{m}\end{array}$ & $\begin{array}{c}\text { HEPA } \\
\text { Filter } \\
\text { Efficiency }(8) \\
\end{array}$ \\
\hline \multirow[t]{4}{*}{$P 4-1$} & 0 & 0.31 & 2.87 & $8.0605 \times 10^{8}$ & \\
\hline & $1 *$ & 0.31 & 2.01 & $3.7865 \times 10^{3}$ & $99.99+$ \\
\hline & $2 *$ & 0.40 & 1.69 & $6.1917 \times 10^{0}$ & $99.98+$ \\
\hline & 3 & - & - & $3.6742 \times 10^{-4}$ & 99.94 \\
\hline \multirow[t]{4}{*}{$P 4-2$} & 0 & 0.37 & 2.46 & $1.4234 \times 10^{9}$ & \\
\hline & $1 * *$ & - & - & $4.9045 \times 10^{3}$ & 99.99+ \\
\hline & 2 & 0.34 & 1.65 & $0.0985 \times 10^{0}$ & 99.99+ \\
\hline & 3 & - & - & $4.3328 \times 10^{-4}$ & 99.50 \\
\hline \multirow[t]{4}{*}{$P 4-3$} & 0 & 0.38 & 2.51 & $3.2595 \times 10^{9}$ & \\
\hline & $i$ & 0.37 & 1.76 & $2.9023 \times 10^{3}$ & $99.99+$ \\
\hline & 2 & 0.36 & 1.68 & $6.8608 \times 10^{-2}$ & $99.99+$ \\
\hline & 3 & - & - & $2.9780 \times 10^{-4}$ & 99.55 \\
\hline \multirow[t]{4}{*}{$P 4-4$} & 0 & 0.34 & 3.00 & $4.0618 \times 10^{9}$ & \\
\hline & 1 & 0.36 & 1.99 & $2.7817 \times 10^{4}$ & $99.99+$ \\
\hline & 2 & 0.34 & 1.89 & $1.4659 \times 10^{-1}$ & $99.99+$ \\
\hline & 3 & - & - & $9.2308 \times 10^{-5}$ & 99.92 \\
\hline \multirow[t]{4}{*}{$P 4-5$} & 0 & 0.66 & 3.28 & $5.2160 \times 10^{9}$ & \\
\hline & 1 & 0.38 & 2.10 & $6.5195 \times 10^{3}$ & $99.99+$ \\
\hline & 2 & 0.39 & 2.09 & $0.9495 \times 10^{-2}$ & $99.99+$ \\
\hline & 3 & - & - & $3.3035 \times 10^{-4}$ & 99.63 \\
\hline \multirow[t]{4}{*}{$P 4-6$} & 0 & 0.48 & 3.76 & $9.1352 \times 10^{9}$ & \\
\hline & 1 & 0.44 & 1.69 & $2.2060 \times 10^{3}$ & $99.99+$ \\
\hline & 2 & 0.42 & 1.66 & $5.0072 \times 10^{-1}$ & $99.99+$ \\
\hline & 3 & - & - & $1.8115 \times 10^{-4}$ & 99.60 \\
\hline \multirow[t]{4}{*}{ P4-7 } & 0 & 0.48 & 2.98 & $4.7413 \times 10^{9}$ & \\
\hline & 1 & 0.47 & 1.96 & $2.6012 \times 10^{4}$ & $99.59+$ \\
\hline & 2 & 0.42 & 1.68 & $2.0763 \times 10^{-1}$ & $99.99+$ \\
\hline & 3 & - & - & $1.9477 \times 10^{-4}$ & 99.89 \\
\hline \multirow[t]{4}{*}{$P 4-8$} & 0 & 0.47 & 3.26 & $5.2885 \times 10^{9}$ & \\
\hline & 1 & 0.48 & 1.70 & $2.1143 \times 10^{4}$ & $99.99+$ \\
\hline & 2 & 0.41 & 1.69 & $1.8053 \times 10^{-1}$ & $99.99+$ \\
\hline & 3 & - & - & $6.2272 \times 10^{-5}$ & 99.96 \\
\hline
\end{tabular}


SUMMARY OF HEPA FILTER EFFICIENCY

HEPA Pu Aerosol Filter amad Ránge Stage

$$
\begin{gathered}
\text { HEPA Filtex } \\
\text { Efficiency }(q) \\
\text { Minimum Avg. Maximum }
\end{gathered}
$$$$
\begin{array}{lllll}
1 & 0.31-0.66 & 99.99+ & 99.99+ & 99.99+ \\
2 & 0.31-0.48 & 99.98+ & 99.99+ & 99.99+ \\
3 & 0.34-0.42 & 99.50 & 99.75 & 99.96
\end{array}
$$

Efficiency of the first and second HEPA filters in series as a function of size was also well within minimum requirements. A computer print-out for Run P4-7 (typical of Runs P4-1 through $\mathrm{P4-8}$ ) is reproduced as Table IV. This shows the HEPA filter efficiency as function of particle size; the combined protection factor (HEPA's $\$ 1$ and \#2) as a furction of particle size for the first two HEPA's: filter efficiency based on gross MF-1 filter samplers upstream and downstream of each HEPA; and the overall protection factors for two or three HEPA's in series. Protection factors for two HEPA's in series ranged from $1.3 \times 10^{9}$ to $1.7 \times 10^{11}$ against aerosols $0.31-0.66 \mu \mathrm{m}$ amad, while for three HEPA's the protection factor ranged from $2.1 \times 10^{12}$ to $4.7 \times 10^{13}$. Table IV shows these protection factors to be $1.97 \times 10^{10}$ and $2.35 \times 10^{13}$. Overall efficiencies based on total Andersen impactor activity agreed quite closely with the overall efficiencies as given by the gross MF-1 filter samplers. Aerosol concentrations for these runs have ranged from $3.3 \times 10^{9} \mathrm{dps} / \mathrm{m}^{3}$ up to approximately $1.7 \times 10^{10} \mathrm{dps} / \mathrm{m}^{3}$. The closer the initial aerosol concentration is to $1.7 \times 10^{10} \mathrm{dps} / \mathrm{m}^{3}$, the fewer are the low level counting problems associated with the sampler downstream of HEPA \#3.

TABLE IV

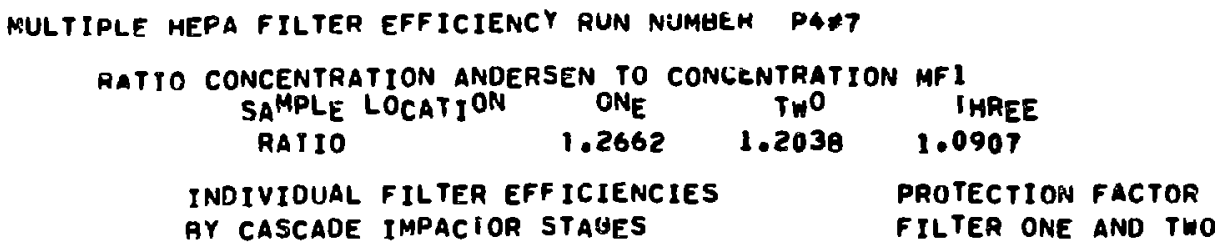

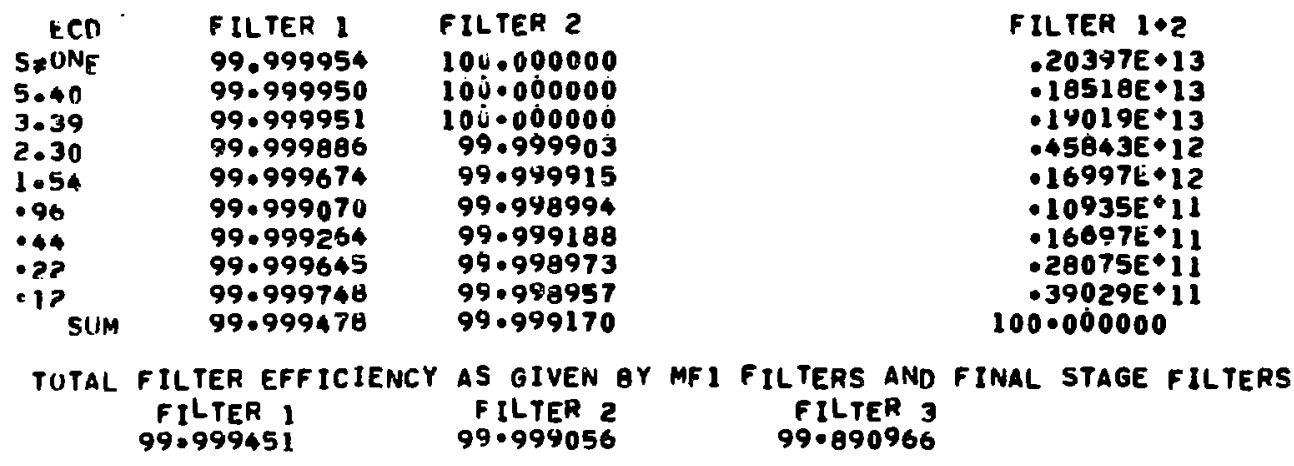

MROTECTION FACTORS AS GIVEN GY FILTER COLLECTIONS

FILTER 1 +2= . $19681 E+11$ FILTEM 1+2+3= .23456E+14 
III. FIELD SAMPLING

A. Spectrum Analysis of Impactor Samples

Field sampling in process ventilation exhaust ducts at Location $00^{1}$ was recently resumed to obtain plutonium particle size data for the two major isotopes by spectrum analysis of impactor samples. Separation of the ${ }^{238} \mathrm{Pu}$ and ${ }^{239} \mathrm{Pu}$ size distribution in the mixture arriving at the final filters is obtained by comparing the alpha peaks of each isotope and applying relative fractions to the gross alpha activity on each impactor stage. Size distribution by isotope can then be expressed as mean percent activity within a size interval or by $\log$ probability representation (activity median aerodynamic diameter and geometric standard deviation) as in earlier reports. Separation of the isotopic content permits (1) better identification of sources, since the isotopes are not normally handied in the same building, and (2) conversion of the amad to either mass median or count median diameter by the Hatch and choate equations. 5

A spectrum analysis system containing a multichannel analyzer and silicon surface barrier detector has been calibrated and its alpha energy resolution optimized for the peaks of ${ }^{238} \mathrm{Pu}(5.5 \mathrm{MeV})$ and ${ }^{239} \mathrm{Pu}(5.1 \mathrm{MeV})$ Full width half maximum (FWHM) resolution at 60 to $65 \mathrm{keV}$ was somewhat poorer than the 28 kev value provided by the manufacturer, but separation of the peaks has been adequate to allow determination of isotopic fractions. Some degradation of alpha peaks has been noted, probably rue to greater self-absorption in larger farticles and absorption in inert material collected on the sample. In cases where degradation of the peaks prohibits integrating to obtain the area under each peak, the isotopic fraction was based on peak height. Though a less reliable technique, use of peak height was based on the assumptions (1) that the presence of an absorbing agent degraded both peaks equally and (2) the spectrum analyzer acquires alpha pulses of both isotopes with equal resolution.
These assumptions are believed valid due to the close proximity of the two alpha energies and general appearance of the peaks observed to date.

A typical set of alpha spectra is shown in Figure 2, where the effective cutoff diameter (ECD) for each impactor stage is also noted. The peak on the left is ${ }^{239} \mathrm{pu}$. At stage 0 , where particles are large, the ${ }^{239} \mathrm{pu}$ peak is rather seriously degraded (probably by self-absorption). while very little ${ }^{238} \mathrm{Pu}$ is present. progressing downward in cutoff diameter in the impactor, the relative amount of ${ }^{238} \mathrm{Pu}$ increases until it dominates the total activity present on stage $5(1.1-2.1 \mu \mathrm{m})$, then diminishes somewhat on the last three stages. In the example shown in Figure 2, the amad and $\sigma_{g}$ of the mixture was $1.62 \mu \mathrm{m}$ and 1.47 , respectively; for ${ }^{239} \mathrm{Pu}$ alone, $1.80 \mu \mathrm{m}$ and 1.63 ; and for ${ }^{238} \mathrm{Pu}$ alone, $1.55 \mu \mathrm{m}$ and 1.37. Neither component departs significantly from the characteristics of the mixture in this instance, but more data will be necessary to develop a clear picture of predominant particle size intervals of individual isotopes. Wide variations in isotopic ratios have been observed, necessitating additional samples and analysis. B. Particle Rebound Effects on Several Impactor plate coatings

In conjunction with the field sampling for isotopic particle size distributions described above, an identical arrangement of two Andersen impactors provided a means for evaluating particle rebound from several impaction surfaces: bare stainless steel, glass fiber filters, vinyl membrane filters, or the Millipore AA membrane filter utilized in the laboratory experiment. In the absence of a known aerosol size distribution, these rebound effects must be measured relative to a reference surface such as the glass fiber filter. Glass fiber filter media has been noted as an effective anti-rebound agent. As reported earlier, ${ }^{4}$ vinyl membrane filters showed no evidence of rebound at normal flow $(1.0 \mathrm{cfm})$ and very little at 
elevated flow $(3.0 \mathrm{cfm})$. However, more precise data are desired for the Millipore AA membrane filter. The data are gathered by sampling with two matched impactor systems from the same location at the same time,but with one impactor coated with reference media. Any difference in amad or percent activity on a given stage could be attibuted to particle rebound from the test media. The experiment is still in early stages of system matching and has produced no reportable data.

IV. EFFECT OF ALPHA RADIATION ON HEPA FILTER MEDIA

Investigation into possible mechanisms of F.EPA filter efficiency loss has continued in the area of radiation damage to glass fiber media by alpha emitters. The postulated efficiency loss is based on particles of high specific activity causing repeated fiber breakage, leading to reduced numbers of fibers which airborne particles must escape in traversing the filter mat.

Alpha radiation can damage glass under certain conditions, particularly noted as silica leached from Pyrex bottles containing acid solutions of alpha-emitting compounds. $6,7,8$ Pyrex and the glass used in some brands of HEPA filters are borosilicate glass. Danage to Pyrex containers appeared as very fine cracks ${ }^{7}$ after exposure to an integrated alpha flux on the order of $10^{20}$ $\alpha / \mathrm{m}^{2}$. Leaching of silica has been observed at exposures as $10 \mathrm{w}$ as $10^{18} \alpha / \mathrm{m}^{2}$. Specific reference to glass fiber damage by alpha radiation was not found.

Assuming the chief mode of failure of glass fibers is by cracking as observed in Pyrex glass surfaces and also assuming the thickness of affected surface exceeds the diameter of glass fibers, breakage depends on the integrated alpha flux in the fiber from a particle of high specific activity in direct contact with the fiber. An estimate of the integrated fiux in a fiber (nominal

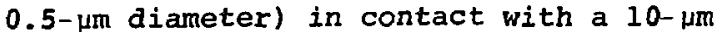

diameter spherical pazticle of ${ }^{238} \mathrm{PuO}_{2}$ for one year was obtained as follows:

Model: A $10 \mu \mathrm{m}$ spherical ${ }^{238} \mathrm{PuO}_{2}$ particle rests in contact with a glass fiber $0.5 \mathrm{um}$ in diameter.

Data: Particle density $1.0 \times 10^{4} \mathrm{~kg} / \mathrm{m}^{3}$ Alpha energy $\left(E_{a}\right) 5.5 \mathrm{MeV}$ specific activity $\left(A_{s}\right) 3.42 \times 10^{20}$ $\min ^{-1} \mathrm{~m}^{-3}$

Alpha range: $11 \mathrm{\mu m}$ in $\mathrm{PuO}_{2}{ }^{9}$ $28.5 \mu \mathrm{m}$ in glass ${ }^{7}$

Assumptions:

(1) All alpha particles emitted inside the 10- $\mu \mathrm{m}$ sphere escape the sphere.

(2) Alpha flux at the particle surface is uniform.

(3) All alpha particles escaping the sphere traverse the fiber linearly transferring energy to the fiber at about $10^{5}$ $\mathrm{ev} / \mu \mathrm{m} .^{7}$

\section{Calculations:}

(1) Particle Emission Rate $A_{p}$

$A_{p}=A_{s} \times$ particle volume $A_{p}=3.42 \times 10^{20} \times$ $\frac{\pi}{6}\left(1.0 \times 10^{-5}\right)^{3}$

$A_{p}=1.8 \times 10^{5} \mathrm{~min}^{-1}$

(2) Alpha Flux at Particle surface $\phi=A_{p} /$ surface area $\phi=\frac{1.8 \times 10^{5}}{I I\left(1.0 \times 10^{-5}\right)^{2}}$ $\phi=5.7 \times 10^{14} \mathrm{~min}^{-1} \mathrm{~m}^{-2}$

(3) Integrated Alpha Bombardment Per Year

$\phi_{I}=\phi x$ time

$\phi_{I}=5.7 \times 10^{14} \times 5.24 \times 10^{5}$ min $\mathrm{yr}^{-1}$

$\phi_{I}=3.0 \times 10^{20} \mathrm{~m}^{-2} \mathrm{yr}^{-1}$

The calculated value of $3 \times 10^{20} \mathrm{~m}^{-2}$ $y^{-1}$ is in the range of observed effects previously noted. Although this result suggests the possibility of alpha damage as a cause of HEPA efficiency loss, the idea of one particle taking one year to break one fiber must be expanded to many particles breaking fibers before a significant efficiency loss could be expected. 
Any further theoretical treatment of this problem appears too difficult and unreliable to undertake in the absence of any experimental data showing a definite loss of efficiency. We propose to retest one or more HEPA filters used in the early stages of the laboratory experiment. These filters were loaded with significant amounts (4 to $6 \mathrm{~g}$ ) of ${ }^{238} \mathrm{PuO}_{2}$ in the experiment and have been stored (under non-flow conditions) for 8 to 10 months. Assuming an aerosol of size characteristics very similar to the original test aerosol can be produced, efficiency can be remeasured and any loss attributed to degradation by alpha bombardment. The filter will then probably be dismantled for close visual inspection of the media and adhesive.

\section{FUTURE WORK}

1. Aerosol generation techniques capable of providing aerosols with amad's near or below $0.1 \mu \mathrm{m}$ will be further investigated.

2. Sampling techniques for determining size characteristics of plutonium aerosols beyond the present capability of the Andersen impactor will be investigated.

3. Several runs will be made to substantiate the present data obtained with 0.3-to 0.5- $\mu \mathrm{m}$ aerosols (amad). Particular emphasis will be placed on increasing activity collected downstream of the third HEPA filter, probably by selection of first and second stage HEPA filters with slightly lower efficiency (still above the quality control criterion of 99.97\%).

4. HEPA filters exposed to ${ }^{238} \mathrm{PuO}_{2}$ particles for 8 to 10 months will be retested with similar aerosols to determine any efficiency loss due to radiation damage.

5. Tests at less than rated flow for these HEPA filters will be performed.

6. Field sampling and spectrum analysis of dual impactor samples will be con-

tinued.

7. Investigation of plutonium particle rebound from impactor stages coated with various materials will be continued.

\section{REFERENCES}

I. H.J. Ettinger, J.C. Elder, and M. Gonzales, "Performance of Multiple HEPA Filters Against Plutonium Aerosols," IA-5349-PR, January I through June 30,1973.

2. H.J. Ettinger, C.I. Fairchild, L.W. Ortiz, and M.I. Tillery, Aerosol Research and Development Related to Health Hazard Analysis, LA-5359-Pr, July 1973.

3. J.N.H. Hu, Envir. Sci. and Tech., 5, 251, 1971 .

4. H.J. Ettinger, J.C. Elder, and M. Gonzales, "Performance of Multiple HEPA Filters Against Plutonium Aerosols." LA-5170-PR, July 1 through December 31, 1972 .

5. T. Hatch and S.P. Choate, Journal of the Franklin Institute, 207, 369, (1529).

6. P.S. Rudolph, Proceedings of the Eighth Symposium on the Art of Glassblowing, 114-123, June 1963.

7. D.G. Tuck, International Journal of Applied Radiation and Isotopes, 15. 49-57, 1964 .

8. D.G. Tuck, Nature, 177, March 3, 1956.

9. G.N. Huffman and C.J. Kershner, Nucl. Appl. Techn.. 9, 434, 1970. 


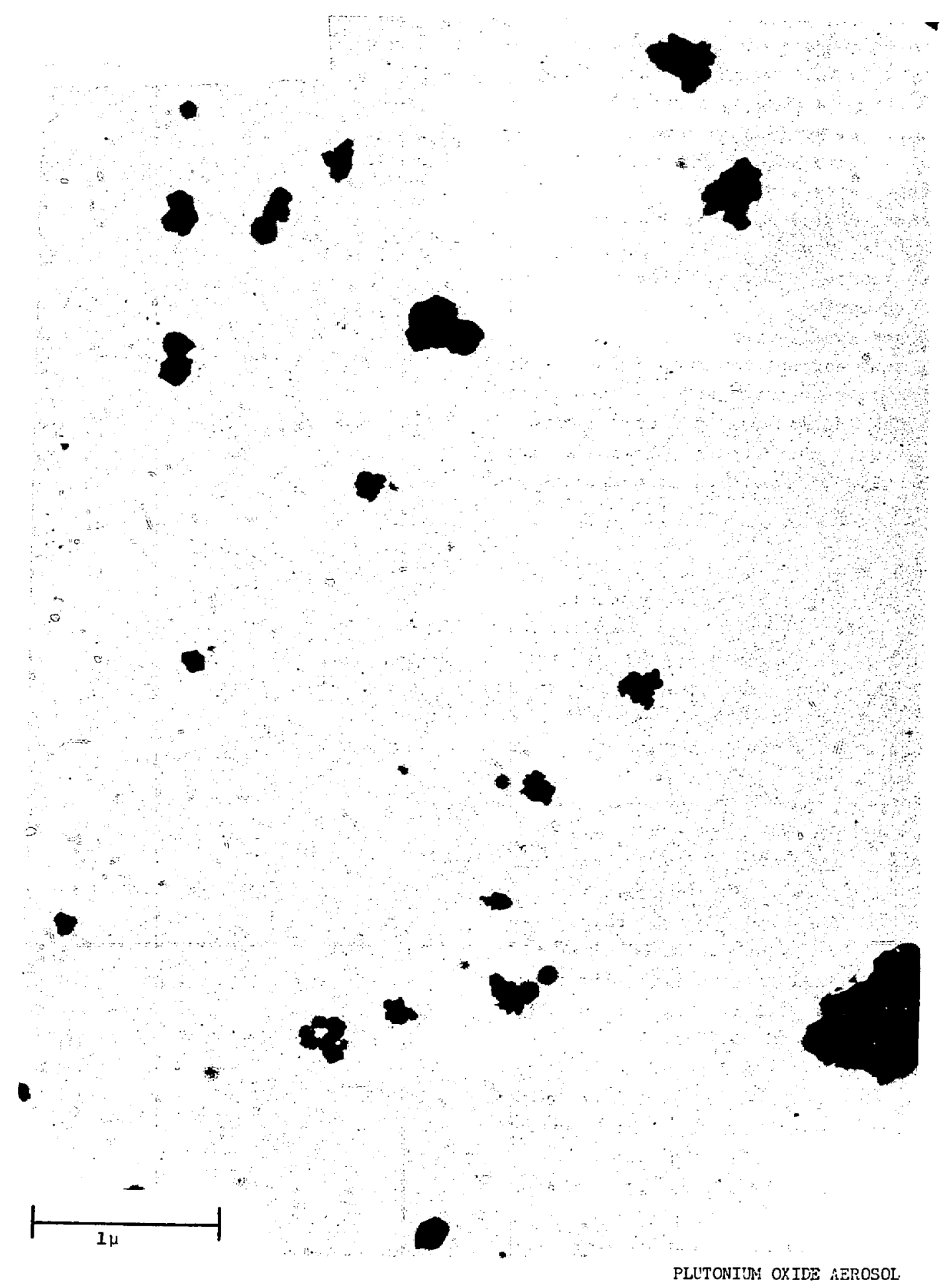

Figure 1. Electronmicrograph of Plutonium Oxide Aerosol. 


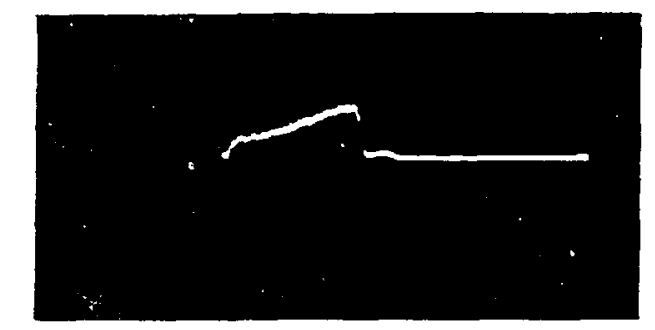

Stage 0

$>11 \mu \mathrm{m}$

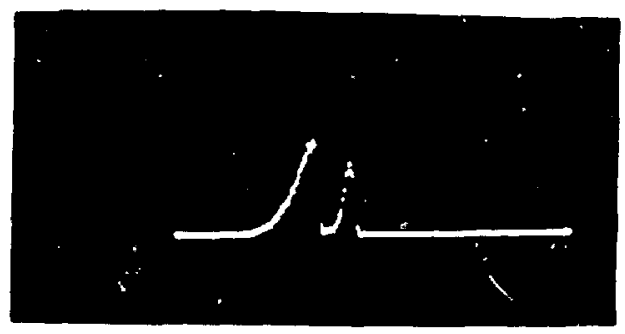

Stage 2

4. 7-7 Lim

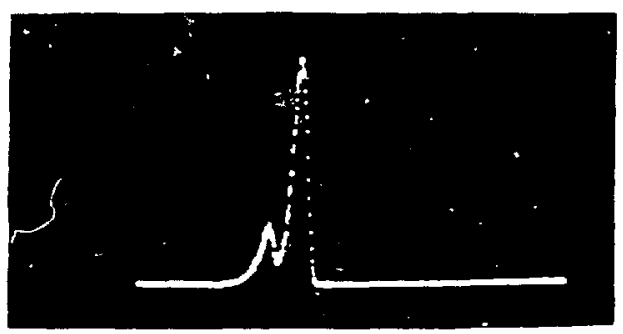

Stage 4

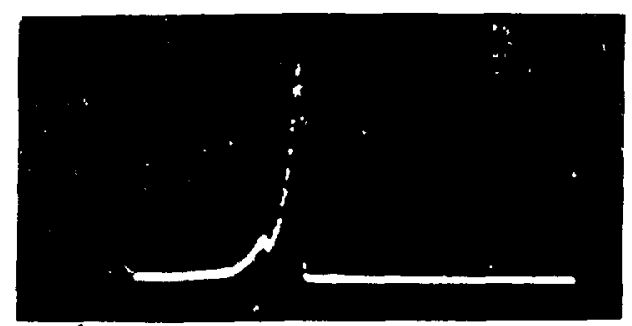

Stage 6
$0.65-1.1 \mathrm{um}$

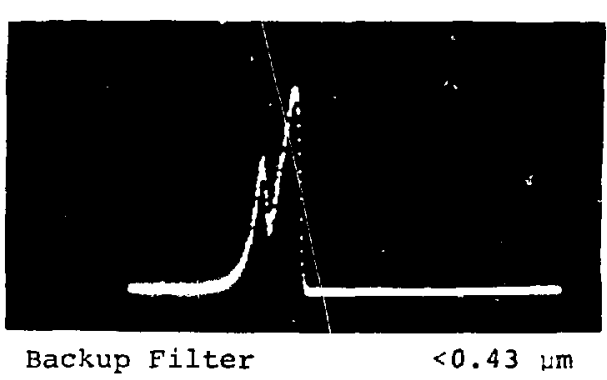

Figure 2. Alpha Spectrurn of Andersen Impactor Samples from Location $00 / 239 \mathrm{Pu}$ Peak on Left).

Stage 7
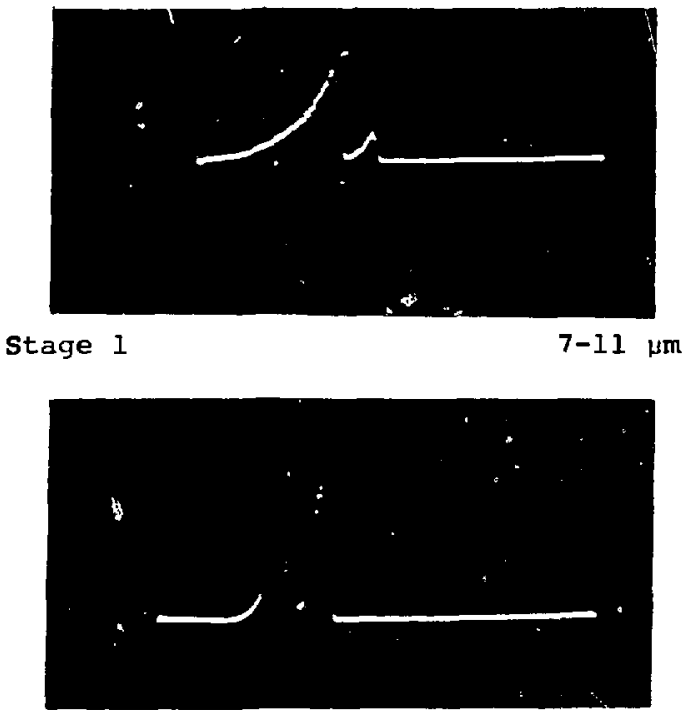

Stage 3

3.3-4.7 um

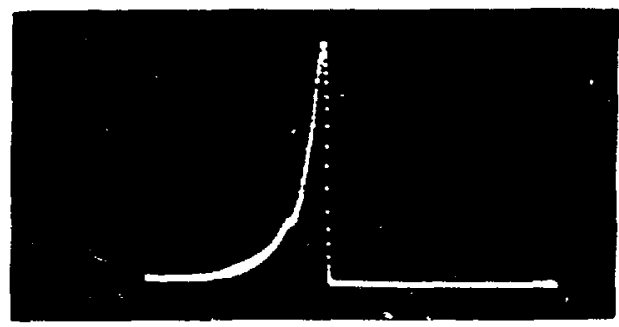

Stage 5

$1.1-2.1 \mathrm{um}$

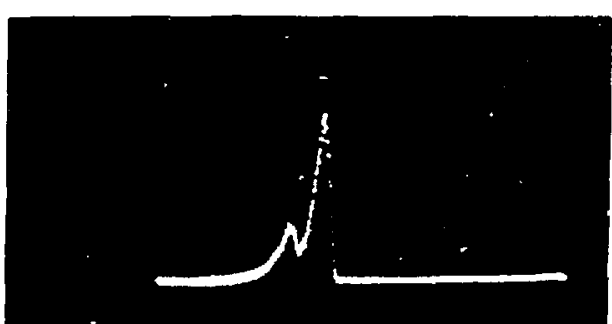

$0.43-0.65 \mu \mathrm{m}$ 